\title{
Vibrating Mesh Nebulizer Compared With Metered-Dose Inhaler in Mechanically Ventilated Subjects
}

\author{
Meagan N Dubosky MSc RRT, Yi-Fan Chen PhD, Mary E Henriksen MSc RRT, and \\ David L Vines MHS RRT FAARC
}

\begin{abstract}
BACKGROUND: The impact of various aerosol delivery devices on patient outcomes during mechanical ventilation is unknown. If one method of delivery results in a higher ventilator-associated pneumonia (VAP) rate than another, multiple patient outcomes may be affected. This study aimed to determine whether there was a difference in VAP occurrence and patient outcomes (days receiving ventilation and in-hospital mortality) between the vibrating mesh nebulizer (AeroNeb Solo) and the metered-dose inhaler (MDI). METHODS: This retrospective study reviewed medical records for all mechanically ventilated, adult patients with an order for aerosol treatment from August 2011 to August 2013. The hospital converted from MDI to vibrating mesh nebulizers in August 2012, and data were gathered 1 y before/after conversion. Excluded were patients with a tracheostomy, patients who were mechanically ventilated for $<24 \mathrm{~h}$, patients who received a combination of nebulizer and MDI treatments, or patients who were re-intubated. RESULTS: Two hundred twenty-eight subjects were included. Forty-eight (21\%) received treatment with an MDI, and 180 (79\%) were treated with the vibrating mesh nebulizer. Descriptive data did not significantly differ for age or APACHE II (Acute Physiology and Chronic Health Evaluation II) scores between the groups but did for sex $(P=.03)$. Difference in median days receiving ventilation for the MDI (5 d, interquartile range 3.0-8.5 d) and the vibrating mesh nebulizer (6 d, interquartile range 4.0-10.0 d) was not statistically significant. No correlation was found between the use of either device and the primary outcomes of VAP and in-hospital mortality. In multivariable logistic regression analysis, the number of days receiving ventilation increased the odds of VAP (odds ratio [OR] 1.3, 95\% CI 1.14-1.49, $P<.001)$ and mortality (OR 1.12, 95\% CI 1.04-1.21, $P=.002)$. Higher APACHE II scores increased the odds of mortality (OR 1.05, 95\% CI 1.001-1.092, $P=.044)$. CONCLUSION: We found no association between an MDI or vibrating mesh nebulizer and our primary outcomes, days receiving ventilation, in-hospital mortality, or VAP, in mechanically ventilated subjects. Key words: metereddose inhalers; nebulizer; ventilators; mechanical; administration; inhalation; patient outcome assessment; APACHE; pneumonia; ventilator-associated. [Respir Care 2017;62(4):391-395. (C) 2017 Daedalus Enterprises]
\end{abstract}

\section{Introduction}

Although the impact on aerosol delivery is considerable, the choice of delivery devices in mechanically ventilated patients is limited. Clinicians can choose from metered-dose inhalers (MDIs), jet nebulizers in several forms, or vibrating

\footnotetext{
Ms Dubosky, Ms Henriksen, and Mr Vines are affiliated with the Department of Cardiopulmonary Sciences, Rush University Medical Center, Chicago, Illinois. Dr Chen is affiliated with the Institute for Health Research and Policy, University of Illinois, Chicago, Illinois.

Ms Dubosky, Ms Henriksen, and Mr Vines presented versions of this paper at the AARC Congress, held November 7-10, 2015, in Tampa, Florida.
}

mesh nebulizers to deliver medications in-line through the ventilator circuit, with each device having its own positive and negative attributes. There are limited data evaluating clinical outcomes related to different aerosol devices.

When MDIs are used in mechanically ventilated patients, optimal delivery requires that the clinician properly 
coordinate MDI actuation with inspiration. Fink and Dhand ${ }^{1}$ conducted a study to test the delivery of aerosol with MDI actuation synchronized with the start of inspiration, $1 \mathrm{~s}$

\section{See the Related Editorial on Page 513}

before inspiration, and at the start of exhalation. They found that the greatest amount of drug was delivered when actuation was synchronized with the start of inspiration. ${ }^{1}$ If not well coordinated; a portion of the delivered dose will be swept into the expiratory limb of the circuit and can reduce the delivered dose by up to $90 \% .^{2}$ However, it still has been suggested that MDIs can be as effective as nebulizers, when used properly. ${ }^{3,4}$ Many hospitals continue to use MDIs for their convenience and perhaps the reduced risk of bacterial contamination. ${ }^{5}$ Cost savings may also be found with the use of a common canister protocol with mechanically ventilated patients without increasing the risk of cross-contamination. ${ }^{6}$

There are additional factors affecting aerosol delivery in mechanically ventilated patients. The presence of the artificial airway alone can significantly reduce the efficiency of an aerosol delivery device. ${ }^{4}$ The type of adapter used to deliver an MDI can impact drug delivery to the patient, such as the use of a chamber spacer resulting in greater drug delivery than an elbow adapter. ${ }^{5}$ The addition of heat and humidity ${ }^{4}$ and bias flow ${ }^{7}$ in the ventilator circuit has been shown to increase aerosol loss.

Aerosol delivery can also be affected by the position of the delivery device in the circuit. A study done by Ari et $\mathrm{al}^{8}$ compared a jet nebulizer, vibrating mesh nebulizer, ultrasonic nebulizer, and pressurized MDI, each placed in 3 locations in the ventilator circuit in both heated, humidified circuits and non-humidified circuits. There were significant changes in aerosol delivery from each device, depending on the humidity and positioning of the device. The vibrating mesh nebulizer, ultrasonic nebulizer, and pressurized MDI provided the highest deposition when placed in the inspiratory limb, $15 \mathrm{~cm}$ from the Y-piece. The jet nebulizer proved to be less efficient, since the continuous flow from the device pushed the aerosol into the expiratory limb except during inspiration. ${ }^{7,8}$ In another study, a jet nebulizer and a vibrating mesh nebulizer were each tested in 2 positions: the dry side of the inspiratory limb before the heater and between the Y-piece and the inspiratory limb. The results showed that both nebulizers delivered more aerosol when placed before the humidifier when bias flow was present. The vibrating mesh nebulizer consistently delivered more drug than the jet

Correspondence: Meagan N Dubosky MSc RRT, Rush Oak Park Hospital, Respiratory Department, Oak Park, IL 60304. E-mail: Meagan_Dubosky@rush.edu.

DOI: $10.4187 /$ respcare. 04823

\section{QUICK LOOK}

\section{Current knowledge}

Respiratory therapists have the option to administer aerosol treatments to a mechanically ventilated patient with a nebulizer or metered-dose inhaler. Current literature does not provide patient outcome data supporting one delivery device over the other.

\section{What this paper contributes to our knowledge}

There was no difference in in-hospital mortality, VAP, or number of ventilator days when comparing treatments with a vibrating mesh nebulizer and metereddose inhaler during mechanical ventilation.

nebulizer. ${ }^{9}$ Although bench testing suggests that more aerosolized drug is delivered, there are limited to no outcome data to demonstrate that vibrating mesh nebulizers reduce length of mechanical ventilation or hospital stay.

The lowering of incidence of hospital-acquired infections is another important initiative in mechanically ventilated patients. Ventilator-associated pneumonia (VAP) has been associated with high mortality rates, increased hospital stay, and significant increases in cost of care. ${ }^{10,11}$ VAP has been linked to the delivery of contaminated aerosols via circuit manipulation and in-line medication delivery. ${ }^{12,13}$ When using an adapter or holding chamber in-line with the ventilator circuit, a port or chamber must be opened, and the circuit must be manipulated. This increases the risk of contamination to the circuit and/or secretions draining back into or around the endotracheal tube. Vibrating mesh nebulizers typically remain inline on the dry side of the humidifier and are not routinely changed, but the cap to the medication cup is routinely opened, which creates a contamination risk. ${ }^{12}$

Although bench testing suggests that more aerosolized drug is delivered, there are limited to no outcome data to demonstrate that vibrating mesh nebulizers reduce the length of mechanical ventilation. The aim of this study is to determine the impact of using an MDI or vibrating mesh nebulizer on the incidence of VAP.

\section{Methods}

This retrospective study was approved by our institutional review board. The electronic medical records for all mechanically ventilated, adult patients with an order for aerosol therapy from August 2011 through August 2013 were reviewed. The hospital, a large academic medical center in the Midwest, had converted from MDI to vibrating mesh nebulizers in August 2012; therefore, data were reviewed $1 \mathrm{y}$ before and after the conversion. This study excluded patients with a tracheostomy who re- 
ceived $<24 \mathrm{~h}$ of invasive mechanical ventilation, those who received a combination of MDI and nebulizer treatments, and patients who were extubated and re-intubated during their hospitalization. The data gathered included the number of days receiving ventilation, in-hospital mortality, and the incidence of VAP. VAP incidence was confirmed by noting ICD-9 997.31 in the subject's electronic medical record, and this diagnosis was confirmed by applying the Centers for Disease Control and Prevention criteria based on imaging, clinical, and laboratory findings (http://www.cdc.gov/nhsn/ PDFs/pscManual/6pscVAPcurrent.pdf. Accessed November $26,2016)$. The chart review also included the subjects' demographics, primary diagnosis code, $\operatorname{drug}(\mathrm{s})$ delivered and dosage of treatment, and the total number of treatments received while subjects were mechanically ventilated.

\section{Statistical Analysis}

The data were summarized by using the frequency with the percentage for categorical variables and the median with the interquartile range for continuous variables. To explore the correlation between covariates and the device MDI versus nebulizer, the chi-square test or Fisher exact test was used for categorical variables, whereas the Wilcoxon rank-sum test was utilized for continuous variables due to skewness of the distributions. Moreover, we fitted logistic regressions to investigate the effect of the device on 2 main outcomes, ventilator-associated pneumonia and mortality, with the Firth penalized likelihood approach as appropriate. Each model adjusted for number of days receiving ventilation, total aerosol medication administrations, APACHE II (Acute Physiology and Chronic Health Evaluation II) score, primary diagnosis code, age, and sex. All of the statistical analyses were conducted by using SAS 9.3 (SAS Institute, Cary, North Carolina). Two-tailed $P$ values of $<.05$ were considered as statistically significant.

\section{Results}

Study staff reviewed the charts of 2,905 patients who were admitted to the hospital's ICUs between August 2011 and August 2013; 2,677 met exclusion criteria and were removed from the sample (Fig. 1). The majority of exclusions included patients who received no aerosol treatments $(n=1,932)$, received $<24$ h of mechanical ventilation $(n=430)$, or received a tracheostomy $(n=177)$. Of the 228 subjects meeting inclusion and avoiding exclusion criteria, 48 subjects (21\%) received treatments with an MDI, and 180 subjects (79\%) were treated with the vibrating mesh nebulizer. Descriptive data (Table 1) did not significantly differ for age, race, or APACHE II scores between the groups but did for sex $(P=.03)$. There were 115 females and 113 males total with 31 females (65\%) and 17 males (35\%) in the MDI group versus 84 females (47\%) and 96 males (53\%) in the vibrating mesh nebulizer group. The difference in median days of in-

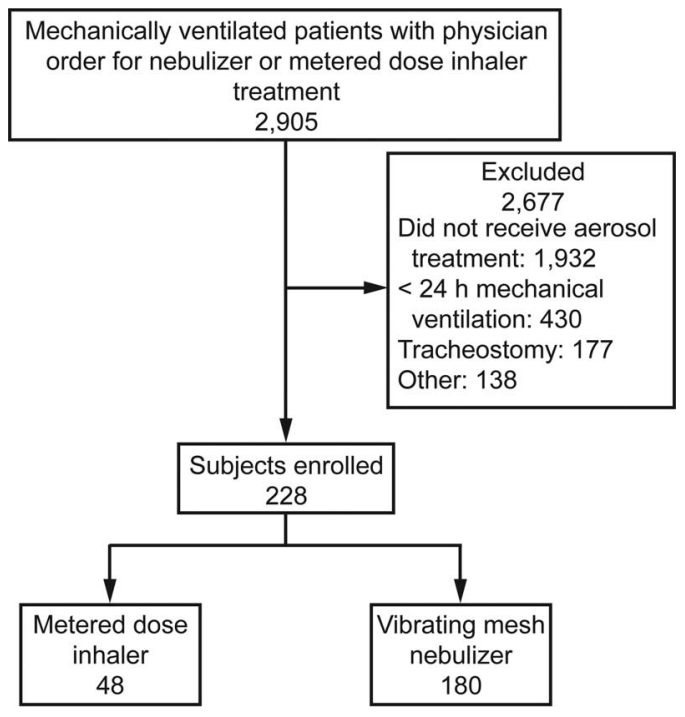

Fig. 1. Flow chart.

vasive mechanical ventilation between the MDI group ( $5 \mathrm{~d}$, interquartile range 3.0-8.5 d) and the vibrating mesh nebulizer group $(6 \mathrm{~d}$, interquartile range $4.0-10.0 \mathrm{~d})$ was not found to be statistically significant. No correlation was found between the use of either device and primary outcomes of VAP or in-hospital mortality.

The medications nebulized via the vibrating mesh nebulizer included a combination of albuterol sulfate, ipratropium bromide, acetylcysteine, racemic epinephrine, budesonide, and levalbuterol. A majority of the vibrating mesh nebulizer treatments $(n=78,43 \%)$ were a combination of albuterol sulfate $(2.5 \mathrm{mg} / 0.5 \mathrm{~mL})$ and ipratropium bromide $(0.02 \%)$. Medications delivered with the MDI were an albuterol sulfate and ipratropium bromide combination $(n=32,67 \%)$, albuterol alone $(n=13,27 \%)$, or ipratropium bromide alone $(n=3,6 \%)$.

In the multivariable logistic regression analysis (Table 2), the days receiving ventilation increased the odds of VAP $(\mathrm{OR}$ $1.3,95 \%$ CI 1.14-1.49, $P<.001)$ and in-hospital mortality (OR 1.12, 95\% CI 1.04-1.21, $P=.002$ ) (Table 3). As shown, the higher APACHE II scores increased the odds of mortality (OR 1.05, 95\% CI 1.001-1.092, $P=.044$ ).

\section{Discussion}

This retrospective study shows no difference in days receiving ventilation or in-hospital mortality for either the MDI or vibrating mesh nebulizer groups. Although others have reported a higher total delivered dose when using a small-volume nebulizer compared with the MDI, ${ }^{14}$ there was no noticeable difference in the number of ventilator days from subjects receiving treatments with a vibrating mesh nebulizer compared with the MDI. This lack of difference could be due to the proper delivery technique used by the medication-administering respiratory therapist, or the MDI doses 
Table 1. Demographics and Characteristics

\begin{tabular}{|c|c|c|c|c|}
\hline Characteristic & Total $(N=228)$ & MDI $(n=48)$ & Nebulizer $(n=180)$ & $P^{*}$ \\
\hline APACHE II, median (IQR) & $17(13-22)$ & $17(13-23)$ & $17(13-22)$ & .67 \\
\hline In-hospital mortality, $n(\%)$ & $76(33)$ & $16(33)$ & $60(33)$ & $>.99$ \\
\hline Age, median (IQR) & $63(53-73)$ & $66(56-75)$ & $62(51-73)$ & .07 \\
\hline Female sex, $n(\%)$ & $115(50)$ & $31(65)$ & $84(47)$ & .03 \\
\hline Race, $n(\%)$ & & & & .28 \\
\hline White & $103(45)$ & $22(46)$ & $81(45)$ & \\
\hline African American & $101(45)$ & $24(50)$ & $77(43)$ & \\
\hline Other & $23(10)$ & $2(4)$ & $21(12)$ & \\
\hline Total treatment administrations, median (IQR) & $7(3-17)$ & $9.50(4-20)$ & $7.00(3-16)$ & .14 \\
\hline Discharge diagnosis, $n(\%)$ & & & & .54 \\
\hline Respiratory & $41(18)$ & $10(21)$ & $31(17)$ & \\
\hline Cardiac/Vascular & $31(14)$ & $4(8)$ & $27(15)$ & \\
\hline Neuro & $55(24)$ & $9(19)$ & $46(26)$ & \\
\hline Sepsis & $27(12)$ & $7(15)$ & $20(11)$ & \\
\hline Other & $74(32)$ & $18(38)$ & $56(31)$ & \\
\hline $\mathrm{VAP}, n(\%)$ & & & & $.72 \dagger$ \\
\hline No & $216(95)$ & $45(94)$ & $171(95)$ & \\
\hline Yes & $12(5)$ & $3(6)$ & $9(5)$ & \\
\hline Days receiving ventilation, median (IQR) d & $6(4-9)$ & $5(3-8.50)$ & $6(4-10)$ & .14 \\
\hline $\begin{array}{l}\text { * For categorical variables, the chi-square test or Fisher exact } \\
\dagger \text { Fisher exact test. } \\
\text { MDI = metered-dose inhaler } \\
\text { APACHE II = Acute Physiology and Chronic Health Evaluati } \\
\text { IQR }=\text { interquartile range } \\
\text { VAP = ventilator-acquired pneumonia }\end{array}$ & . For continuous variabl & xon rank-sum test w: & & \\
\hline
\end{tabular}

Table 2. Logistic Regression to Assess Factors Impacting the Development of Ventilator-Acquired Pneumonia

\begin{tabular}{lcc}
\hline \hline \multicolumn{1}{c}{ Effect } & Odds Ratio & \multicolumn{1}{c}{$95 \%$ CI } \\
\hline Device (MDI vs nebulizer) & 2.89 & $0.67-12.40$ \\
Days receiving ventilation & 1.31 & $1.14-1.49$ \\
Total treatment administrations & 0.98 & $0.95-1.01$ \\
APACHE II & 0.99 & $0.90-1.10$ \\
Age & 1.01 & $0.97-1.05$ \\
Sex (female vs male) & 1.93 & $0.53-7.04$
\end{tabular}

$\overline{\mathrm{MDI}}=$ metered-dose inhaler

APACHE II = Acute Physiology and Chronic Health Evaluation II

ordered were adequate or adjusted to response. Bench work focusing on drug delivery noted similar results between the 2 devices used in our study, which may help to explain the similar outcomes found in our study. ${ }^{7,8}$

There was also no difference noted in the incidence of VAP between medication delivery devices. Dhand and Guntur $^{5}$ noted that MDIs reduce the chance of bacterial contamination. The vibrating mesh nebulizer is usually placed back at the dry inlet of the humidifier, so there is less manipulation of the breathing circuit, which may lower the risk of VAP. Our institution also uses a ventilator bundle, and oral care with chlorhexidine is provided every
Table 3. Logistic Regression to Assess Factors Impacting Mortality

\begin{tabular}{lcc}
\hline \multicolumn{1}{c}{ Effect } & Point Estimate & $95 \% \mathrm{CI}$ \\
\hline Device (MDI vs nebulizer) & 1.11 & $0.52-2.37$ \\
Days on ventilator & 1.12 & $1.04-1.21$ \\
Total treatment administrations & 1.00 & $0.98-1.02$ \\
APACHE II & 1.05 & $1.00-1.09$ \\
Age & 0.99 & $0.98-1.01$ \\
Sex (female vs male) & 0.92 & $0.50-1.68$
\end{tabular}

MDI $=$ metered-dose inhaler

APACHE II = Acute Physiology and Chronic Health Evaluation II

$4 \mathrm{~h}$ to lower the incidence of VAP. Seeing no difference and low incidence of VAP between the 2 devices could reflect the clean technique used by the respiratory therapists and the institution's adherence to VAP prevention.

The notable increase in incidence of VAP with the higher number of ventilator days is to be expected, considering the known higher risk of VAP for long-term ventilated patients. ${ }^{15}$ It should be noted that the definition for VAP used in this study is no longer supported by the Centers for Disease Control and Prevention. This study should be repeated using the current tiered system of ventilator-associated events (ventilator-associated condition, infection-related ventilator-associated complication, and VAP). Available evidence suggests 
that ventilator-associated condition and infection-related ventilator-associated complication are associated with poor outcomes, whereas VAP is not. The higher APACHE II scores showing an increase in mortality among the subjects is also logical. ${ }^{16}$ As expected, patients with higher APACHE II scores, attributed to more severe illness, have a higher mortality rate..$^{15,16}$

Our study might be underpowered for the outcomes of interest. Following exclusions, there were $<50$ subjects in the MDI group. The odds ratio for VAP was 2.89 , which might be clinically important, but it is not significant, probably due to the small number of subjects who received the MDI. This factor makes it difficult to exclude the possibility that VAP was greater with the MDI.

The study was limited because it was retrospective and lacked randomization. Although the data were retrospectively collected and a sex difference exists, there were no significant differences in severity of illness, age, or total number of treatments administered between the 2 groups. There were other limitations in that we are uncertain how the aerosol treatments were actually given. There were department policies in place directing the therapist to deliver the MDI through an adapter 6 inches before the Y-piece on the inspiratory limb of the circuit and to deliver the puffs at the start of inspiration. The vibrating mesh nebulizer should have been placed on the dry side of the humidifier and run until empty per hospital protocol. Both the vibrating mesh nebulizer and MDI adapter should have remained in the circuit. Additional limitations include the fact that the total dosing of albuterol was not controlled; nor was the number of medications delivered. These medications are typically adjusted to desired effect. We are unaware whether the aerosol treatments were or were not indicated or of the effect the bronchodilators had on airway mechanics. An institutional expectation was that therapists discuss discontinuing therapy with the medical providers when it is not indicated. We are unaware whether all aerosol treatments were indicated or whether the effect would have bearing on the development of VAP. ${ }^{11}$ VAP would more likely result from contamination of the delivery device in the case of the nebulizer or manipulation of the circuit, resulting in contaminated fluids draining back into or around the airways.

\section{Conclusions}

This retrospective study showed no difference in number of days receiving mechanical ventilation, in-hospital mortal- ity, or VAP between MDI and vibrating mesh nebulizer use to deliver medicated aerosols in the acute-care setting.

\section{ACKNOWLEDGMENTS}

We are thankful to our colleagues who contributed to this study, including Stanley Letowski, Allison Anderson, Jacob Burd, Darpan Amin, Shannon Sims, and David L Bowton.

\section{REFERENCES}

1. Fink JB, Dhand R. Laboratory evaluation of metered-dose inhalers with models that simulate interaction with the patient. Respir Care Clin N Am 2001;7(2):303-317, vi-vii.

2. Fink JB, Rubin BK. Problems with inhaler use: a call for improved clinician and patient education. Respir Care 2005;50(10):1360-1374, discussion 1374-1375.

3. Fink JB. Aerosol device selection: evidence to practice. Respir Care 2000;45(7):874-885.

4. Duarte AG. Inhaled bronchodilator administration during mechanical ventilation. Respir Care 2004;49(6):623-634.

5. Dhand R, Guntur VP. How best to deliver aerosol medications to mechanically ventilated patients. Clin Chest Med 2008;29(2):277-296, vi.

6. O'Malley CA. Device Cleaning and infection control in aerosol therapy. Respir Care 2015;60(6):917-927, discussion 928-930.

7. Miller DD, Amin MM, Palmer LB, Shah AR, Smaldone GC. Aerosol delivery and modern mechanical ventilation: in vitro/in vivo evaluation. Am J Respir Crit Care Med 2003;168(10):1205-1209.

8. Ari A, Areabi H, Fink JB. Evaluation of aerosol generator devices at 3 locations in humidified and non-humidified circuits during adult mechanical ventilation. Respir Care 2010;55(7):837-844.

9. Ari A, Atalay OT, Harwood R, Sheard MM, Aljamhan EA, Fink JB. Influence of nebulizer type, position, and bias flow on aerosol drug delivery in simulated pediatric and adult lung models during mechanical ventilation. Respir Care 2010;55(7):845-851.

10. Guillamet CV, Kollef MH. Ventilator associated pneumonia in the ICU: where has it gone? Curr Opin Pulm Med 2015;21(3):226-231.

11. Kollef MH. Prevention of hospital-associated pneumonia and ventilator-associated pneumonia. Crit Care Med 2004;32(6):1396-1405.

12. Hess DR, Kallstrom TJ, Mottram CD, Myers TR, Sorenson HM, Vines DL, American Association for Respiratory Care. Care of the ventilator circuit and its relation to ventilator-associated pneumonia. Respir Care 2003;48(9):869-879.

13. Isakow W, Kollef MH. Preventing ventilator-associated pneumonia: an evidence-based approach of modifiable risk factors. Semin Respir Crit Care Med 2006;27(1):5-17.

14. Marik P, Hogan J, Krikorian J. A comparison of bronchodilator therapy delivered by nebulization and metered-dose inhaler in mechanically ventilated patients. Chest 1999;115(6):1653-1657.

15. Trouillet JL, Chastre J, Vuagnat A, Joly-Guillou ML, Combaux D, Dombret MC, Gilbert C. Ventilator-associated pneumonia caused by potentially drug-resistant bacteria. Am J Respir Crit Care Med 1998;157(2):531-539.

16. Del Bufalo C, Morelli A, Bassein L, Fasano L, Quarta CC, Pacilli AM, Gunella G. Severity scores in respiratory intensive care: APACHE II predicted mortality better than SAPS II. Respir Care 1995;40(10):1042-1047.

This article is approved for Continuing Respiratory Care Education credit. For information and to obtain your CRCE

(free to AARC members) visit

www.rcjournal.com

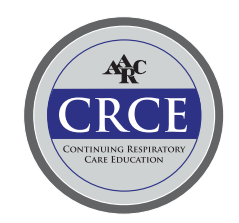

